\title{
TESTE DE RAIOS X NA AVALIAÇÃO DA QUALIDADE DE SEMENTES DE ABÓBORA ${ }^{1}$.
}

\author{
MARIA LAENE MOREIRA DE CARVALHO ${ }^{2}$, CLAUDIA DENISE DA SILVA ${ }^{3}$, LUCIANA MAGDA DE OLIVEIRA4, \\ DÉBORA GABRIELA SILVA ${ }^{5}$, CARLA MASSIMO CALDEIRA ${ }^{6}$
}

\begin{abstract}
RESUMO - As sementes de cucurbitáceas apresentam tegumento espesso, o que dificulta a visualização de sua morfologia interna. Para avaliar a eficiência da utilização do teste de raios X na detecção de danos internos e na avaliação da qualidade de sementes de abóbora, foram utilizados dez lotes de diferentes níveis de qualidade. As sementes foram submetidas às intensidades de 40, 45 e $50 \mathrm{kV}$ por 20, 25 e 30 segundos em aparelho de raios X Faxitron HP modelo 43855A. Definidos a intensidade e o tempo de exposição que proporcionaram melhor visualização das estruturas internas das sementes, o teste de raios $\mathrm{X}$ foi realizado em quatro repetições de 100 sementes, por lote, para determinar a porcentagem de sementes vazias, cheias e translúcidas. Os sete lotes que apresentaram sementes das três categorias, em quantidade suficiente, foram submetidos ao teste de germinação para avaliação da influência da morfologia interna na qualidade fisiológica. Em uma segunda fase, as sementes dos outros três lotes foram divididas, sendo uma fração radiografada e outra não e ambas armazenados por um ano. O teste de raios X realizado na intensidade de $45 \mathrm{kV}$ por 25 segundos é eficiente para identificar danos internos em sementes de abóbora, que afetam negativamente sua qualidade fisiológica. A exposição das sementes a essa radiação não afeta a sua qualidade fisiológica, avaliada mesmo após seu armazenamento pelo período de um ano.
\end{abstract}

Termos para indexação: morfologia interna, qualidade fisiológica, Cucurbita moschata

\section{X-RAY TEST TO EVALUATE PUMPKIN SEED QUALITY}

\begin{abstract}
Cucurbits seeds have a thick seed coat that makes it difficult to view their internal morphology. To evaluate the efficiency of the X-ray test to detect internal damage and to evaluate the pumpkin seed quality, ten lots with different quality levels were used. Seeds were submitted to radiation intensity of 40, 45 and $50 \mathrm{kV}$ and exposition times of 20, 25 and 30 seconds, in a Faxitron HP model 43855A X-ray equipment. After defining the exposure time and radiation intensity that provided the best visualization of seed internal structures, the X-ray test was carried out in four repetitions of 100 seeds, by lot, to determine the percentage of empty seeds, full seeds and translucent
\end{abstract}

${ }^{1}$ Submetido em 28/10/2008. Aceito para publicação, 20/01/2009.

${ }^{2}$ Eng. Agrônoma, Dr., Professora, Depto de Fitotecnia, Universidade Federal de Lavras, 37200-000, Lavras/MG, mlaenemc@ufla.com.br

${ }^{3}$ Eng. Agrônoma, Mestranda em Agronomia (Produção e Tecnologia de Sementes), Dept. de Produção Vegetal, Universidade Estadual Paulista, 14884-900, Jaboticabal/SP, claudiadenises@yahoo.com.br

${ }^{4}$ Eng. Florestal, Dr, Professora, Depto de Eng. Florestal, Universidade do
Estado de Santa Catarina, Centro de Ciências Agroveterinárias, 88520-000, Lages/SC, luciana@cav.udesc.br

${ }^{5}$ Graduanda em Agronomia, Universidade Federal de Lavras, 37200-000, Lavras/MG debora-gabi@hotmail.com

${ }^{6}$ Eng. Agrônoma , Mestranda em Fitotecnia, Depto de Fitotecnia, Universidade Federal de Lavras, 37200-000, Lavras/MG, carlaufla@ yahoo.com.br 
seeds. Seed lots that showed these three categories in sufficient quantities (seven), were submitted to the germination test to evaluate the influence of internal morphology on physiological quality. In a second phase, the seeds of another three lots were divided, one part was radiographed, theother was not radiographed, and both were stored for one year. The X-ray test conducted with $45 \mathrm{kV}$ radiation intensity of for 25 seconds was effective to identify internal damage in pumpkin seeds that negatively affected their physiological quality. The seed exposure to radiation did not affect the physiological quality, even after storage for one year.

Index terms: internal morphology, physiological quality, cucurbita moschata

\section{INTRODUÇÃO}

As cucurbitáceas, com cerca de 90 gêneros e mais de 800 espécies, constituem uma das mais importantes famílias de plantas cultivadas pelo homem. Dentre as espécies de maior expressão econômica merecem destaque a abóbora (Cucurbita moschata), abobrinha (Cucurbita pepo), melão (Cucumis melo L), melancia (Citrullus vulgaris) e pepino (Cucumis sativus $\mathrm{L}$ ).

Os frutos de abóbora são utilizados diretamente na alimentação humana, sendo fonte nutritiva à população carente do centro-sul e nordeste brasileiro, como também são empregados na alimentação animal, na fabricação de doces e de sopas para recém-nascidos. São ricos em vitamina A, vitaminas do complexo $\mathrm{B}$, cálcio, fósforo, silício, magnésio, cloro, ferro, potássio e sódio, com poucas calorias e de fácil digestão (Silva, 2006).

Para a produção comercial de frutos de abóbora, a utilização de sementes de alta qualidade é fator fundamental. Baixas produtividades geralmente estão associadas, dentre outros fatores, a utilização de lotes de sementes com baixa germinação, reduzido vigor e alta contaminação por patógenos. Na cultura da abóbora é freqüente a ocorrência de sementes vazias e danificadas morfologicamente, sendo as últimas de difícil separação e identificação devido ao tipo de beneficiamento que sofrem, também, pela presença de tegumento duro, o qual impede a visualização do embrião sem danificar as sementes.

Técnicas simples e rápidas que forneçam com precisão, informações a respeito de sementes de abóbora podem contribuir para a seleção de lotes em programas de controle da qualidade e, consequentemente, aumentar a eficiência do sistema de produção. Neste contexto, o uso do teste de raios X, técnica recomendada pela ISTA (2004), é de grande valia para um grande número de espécies, por ser um teste não destrutivo, rápido e com aplicação direta na identificação de danos internos de sementes e na seleção de lotes de melhor qualidade.

Apesar dos raios $\mathrm{X}$ serem potencialmente prejudiciais, causando mutações em sementes, a pequena dose de radiação, usada durante o teste para avaliação da qualidade das sementes, não causa danos a maioria das espécies (Carvalho \& Oliveira, 2006). No entanto, não existem informações sobre o efeito latente da radiação em sementes após o armazenamento.

Diante do exposto, este trabalho foi realizado com $\mathrm{o}$ intuito de avaliar a eficiência do teste de raios $\mathrm{X}$ na identificação de danos em sementes de abóbora e sua relação com a germinação, bem como a influência da radiação, a qual as sementes são submetidas, na sua qualidade fisiológica antes e após armazenamento.

\section{MATERIAL E MÉTODOS}

A pesquisa foi realizada no Laboratório de Análises de Sementes do Departamento de Agricultura da Universidade Federal de Lavras, com dez lotes de sementes de abóbora e dividida em dois experimentos.

O grau de umidade dos lotes foi determinado, em quatro gramas de sementes, pelo método de estufa a $105^{\circ} \mathrm{C}$ durante 24 horas, conforme metodologia das Regras para Análises de Sementes (Brasil, 1992), antes e após um ano de armazenamento.

Para determinar a metodologia ideal para realização do teste de raios $\mathrm{X}$ em sementes de abóbora, amostras de 100 sementes de cada lote foram submetidas às intensidades de 40,45 e $50 \mathrm{kV}$ por 20,25 e 30 segundos. As sementes foram arranjadas em placas de isopor com $1 \mathrm{~cm}$ de espessura sobre filme MR 2000-1 Kodak e submetidas à radiação a 
uma distância de $35 \mathrm{~cm}$ da fonte, em aparelho Faxitron HP, modelo 43855A.

Definidos a intensidade e o tempo de exposição que proporcionaram melhor visualização das estruturas internas das sementes, quatro repetições de 100 sementes, por lote, foram submetidas ao teste de raios $\mathrm{X}$ e, de acordo com a morfologia interna visualizada na radiografia, as sementes foram classificadas em vazias (sem embrião), cheias (100\% do embrião desenvolvido e claro), translúcidas (100\% do embrião desenvolvido e escurecido) e danificadas (Figura 1). Os lotes que apresentaram porcentagens de sementes danificadas e translúcidas acima de $10 \%$, foram divididos em três sub-lotes mais a testemunha para a realização do experimento referente à avaliação da influência da morfologia interna na qualidade das sementes (Experimento 1). Os demais lotes foram utilizados no experimento referente ao efeito da radiação na qualidade das sementes antes e após armazenamento (Experimento 2).

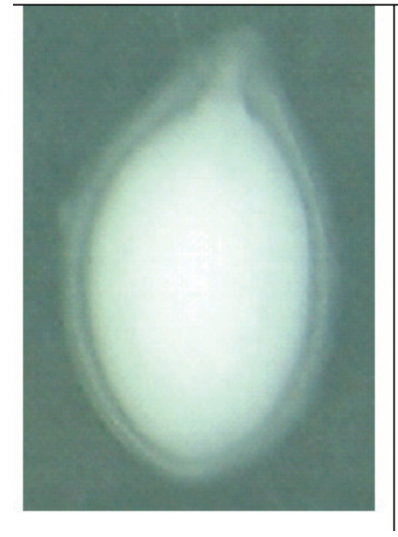

Semente Cheia

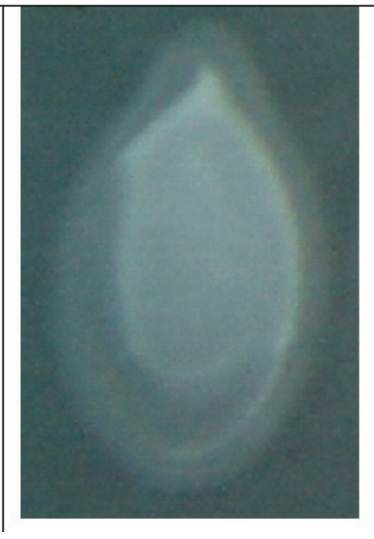

Semente Danificada

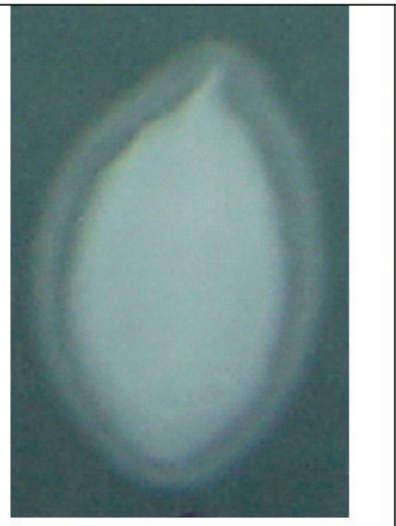

Semente Translúcida

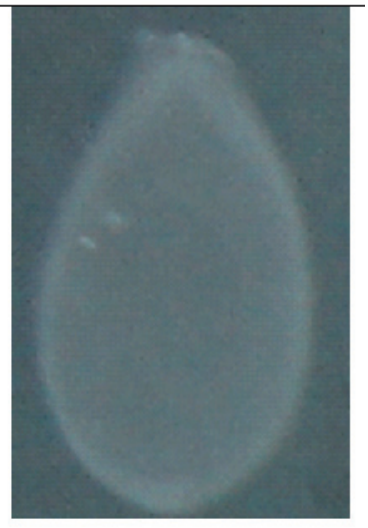

Semente Vazia

FIGURA 1. Imagens radiográficas de sementes de abóbora (Cucurbita moschata).

\section{Experimento 1}

Para a avaliação da influência da morfologia interna na germinação das sementes, cada um dos sete lotes foi dividido em três sub-lotes de acordo com as características de sua morfologia interna visualizada na radiografia em: Sementes Cheias (C), Sementes Danificadas (D) e Sementes Translúcidas (T). Quatro repetições de dez sementes, de cada sub-lote e da testemunha (sementes não radiografadas) foram submetidas ao teste de germinação e avaliadas individualmente.

O teste de germinação foi conduzido em substrato papel tipo Germitest, umedecido com água destilada na proporção de 2,5 vezes a massa do papel, em germinador a $25^{\circ} \mathrm{C}$. As avaliações foram realizadas ao quarto (Primeira Contagem de Germinação) e oitavo dias após a instalação do teste, segundo as Regras para Análise de Sementes (Brasil, 1992).

O delineamento experimental utilizado foi o inteiramente casualizado, em esquema fatorial 7 x 4 ( 7 lotes $\times 4$ sub-lotes) e os dados foram submetidos à análise de variância e ao teste de Tukey a $5 \%$ de probabilidade.

\section{Experimento 2}

Para avaliar a influência da radiação na qualidade das sementes antes e após armazenamento, três lotes de sementes de abóbora foram divididos, sendo uma fração de sementes não radiografadas, e outra radiografada na intensidade de 45 $\mathrm{kV}$ por 25 segundos. As sementes foram armazenadas em embalagens aluminizadas por um período de um ano em câmara fria a $10^{\circ} \mathrm{C}$ e $50 \%$ de umidade relativa do ar.

A qualidade das sementes foi avaliada, antes e após o armazenamento, por meio dos testes de germinação e emergência. O teste de germinação foi conduzido em substrato papel toalha tipo Germitest umedecido com água destilada na proporção de 2,5 vezes a massa do papel, em germinador a temperatura de $25^{\circ} \mathrm{C}$ constante. As contagens foram realizadas ao quarto (Primeira Contagem de Germinação) e ao oitavo dia após a instalação do teste (Brasil, 1992).

$\mathrm{O}$ teste de emergência foi conduzido em bandejas com substrato terra e areia na proporção 1:1, umedecido até $60 \%$ de sua capacidade de saturação. As sementes foram mantidas sob luz constante a uma temperatura de $25^{\circ} \mathrm{C}$, sendo a 
contagem efetuada aos 14 dias.

Foram utilizadas quatro repetições de 50 sementes em cada teste. $\mathrm{O}$ delineamento experimental utilizado foi o inteiramente casualizado em esquema fatorial $2 \times 2$ ( 2 épocas x 2 sub-lotes) e os dados foram submetidos à análise de variância e ao teste de Tukey a 5\% de probabilidade.

\section{RESULTADOS E DISCUSSÃO}

As sementes dos 10 lotes de abóbora estavam com teor de água uniforme em torno de $8 \%$ e $7 \%$ por ocasião da realização dos experimentos antes e após o armazenamento, respectivamente. Essa baixa umidade favorece a visualização das estruturas das sementes durante a análise radiográfica, pois de acordo com Simak (1991), a umidade das sementes influencia a densidade ótica, ou seja, quanto menor a umidade das sementes, maior a densidade ótica, o que possibilita uma maior diferenciação das estruturas das sementes visualizadas nas radiografias.

Os tempos e as intensidades de radiação testados possibilitaram a visualização das estruturas internas das sementes de abóbora de todos os lotes; no entanto, o tempo de 25 segundos e a intensidade de $45 \mathrm{kV}$ permitiram uma visualização mais nítida, o que facilitou a categorização dos defeitos. O tempo de exposição e a intensidade de radiação ideal para a realização do teste de raios $\mathrm{X}$ dependem do aparelho utilizado, do comprimento de onda da radiação, da composição química, espessura e densidade das sementes, que variam de acordo com a espécie (Bino et al., 1993). Argerich \& Bradford (1989), em trabalho realizado com sementes de tomate em aparelho Faxitron, Hewlett-Packard (modelo 43804) Paper M2, concluíram que $15 \mathrm{kV}$ e 2.45 minutos seriam adequados para a realização do teste de raios $\mathrm{X}$; já Van der Burg et al. (1994) trabalhando com a mesma espécie em aparelho Faxitron, Hewlett-Packard (modelo 43805N) determinaram $10 \mathrm{kV}$ e 5 minutos como mais adequados.

Por meio da análise radiográfica foi observado que, dos 10 lotes utilizados, sete lotes possuíam mais que 10\% de sementes danificadas e translúcidas e foram encontradas sementes vazias em apenas dois lotes. A maioria das sementes radiografadas foi considerada cheia, sendo que sua ocorrência variou de 54,75 a $89 \%$ nos diferentes lotes (Tabela 1).

Os lotes $1,3,4,5,7,8$ e 10 foram utilizados no experimento 1 e o restante dos lotes no experimento 2 .

\section{Experimento 1}

De acordo com os resultados apresentados na tabela 2, a velocidade de germinação das sementes cheias de todos os lotes, avaliada pela primeira contagem de germinação, foi superior em relação ao sub-lote de sementes danificadas. Para os lotes $7 \mathrm{e} 8$ as sementes cheias germinaram mais rapidamente que as sementes translúcidas e que a testemunha.

TABELA1. Porcentagem de sementes cheias, danificadas, translúcidas e vazias em lotes de sementes de abóbora (Cucurbita moschata).

\begin{tabular}{ccccc}
\hline Lote & $\begin{array}{c}\text { Sementes } \\
\text { Cheias }\end{array}$ & $\begin{array}{c}\text { Sementes } \\
\text { danificadas }\end{array}$ & $\begin{array}{c}\text { Sementes } \\
\text { translúcidas }\end{array}$ & $\begin{array}{c}\text { Sementes } \\
\text { vazias }\end{array}$ \\
\hline 1 & 74 & 13 & 12 & 0 \\
2 & 84 & 6 & 8 & 0 \\
3 & 70 & 16 & 13 & 0 \\
4 & 80 & 10 & 10 & 0 \\
5 & 69 & 12 & 16 & 1 \\
6 & 84 & 7 & 8 & 0 \\
7 & 78 & 10 & 10 & 0 \\
8 & 68 & 13 & 18 & 0 \\
9 & 89 & 4 & 6 & 0 \\
10 & 54 & 19 & 25 & 0 \\
\hline
\end{tabular}

TABELA 2. Resultados do teste de primeira contagem de germinação dos diferentes sub-lotes de sementes de abóbora (Cucurbita moschata). (C - Sementes Cheias, T - Sementes Translúcidas e D - Sementes Danificadas).

\begin{tabular}{|c|c|c|c|c|}
\hline \multirow{2}{*}{ LOTE } & \multicolumn{4}{|c|}{ SUB-LOTES } \\
\hline & Testemunha & $\mathrm{C}$ & $\mathrm{T}$ & $\mathrm{D}$ \\
\hline 1 & 87 a $A B$ & 92 a $A$ & 85 a $\mathrm{AB}$ & 65 a B \\
\hline 3 & $75 \mathrm{ab} A$ & 87 a A & 85 a A & $35 \mathrm{bc} B$ \\
\hline 4 & $70 \mathrm{ab} A B$ & 95 a A & $70 \mathrm{ab} A B$ & $62 \mathrm{ab} B$ \\
\hline 5 & $65 \mathrm{ab} \mathrm{BC}$ & 100 a A & 87 a $\mathrm{AB}$ & 45 abc C \\
\hline 7 & $55 \mathrm{~b} \mathrm{~B}$ & 87 a $\mathrm{A}$ & $55 \mathrm{~b} \mathrm{~B}$ & $27 \mathrm{c} \mathrm{C}$ \\
\hline 8 & $60 \mathrm{ab} B$ & 95 a $\mathrm{A}$ & $50 \mathrm{~b} \mathrm{~B}$ & $55 \mathrm{abc} \mathrm{B}$ \\
\hline 10 & $77 \mathrm{ab} \mathrm{AB}$ & 100 a A & $95 \mathrm{a} A B$ & 72 a B \\
\hline
\end{tabular}

* Médias seguidas pela mesma letra minúscula na coluna e maiúscula na linha não diferem entre si pelo teste de Tukey a $5 \%$ de probabilidade. 
O sub-lote de sementes cheias de apenas três lotes foram superiores em relação a testemunha, mas apesar de não haver diferenças estatísticas para os demais lotes o número de plântulas normais foi inferior para a testemunha em relação as sementes cheias.

As sementes cheias de todos os lotes apresentaram qualidade fisiológica semelhante, sendo que os demais sub- lotes apresentavam dois níveis de qualidade.

Pela análise dos resultados do teste de germinação (Tabela 3) exceto para um lote, a germinação das sementes cheias foi superior a das sementes danificadas. O sub-lote de sementes cheias resultaram em $100 \%$ de plântulas normais para quatro lotes e os demais apresentaram germinação maior ou igual a $95 \%$.

TABELA 3. Resultados do teste de germinação dos diferentes sub-lotes de sementes de abóbora (Cucurbita moschata). (C- Sementes Cheias, T- Sementes Translúcidas e D- Sementes Danificadas).

\begin{tabular}{ccrll}
\hline \multirow{2}{*}{ LOTE } & \multicolumn{4}{c}{ SUB LOTES } \\
\cline { 2 - 5 } & Testemunha $(\%)$ & $\mathrm{C}(\%)$ & $\mathrm{T}(\%)$ & $\mathrm{D}(\%)$ \\
\hline 1 & $95 \mathrm{a} \mathrm{AB}$ & $100 \mathrm{a} \mathrm{A}$ & $95 \mathrm{a} \mathrm{AB}$ & $75 \mathrm{ab} \mathrm{B}$ \\
3 & $90 \mathrm{ab} \mathrm{A}$ & $100 \mathrm{a} \mathrm{A}$ & $90 \mathrm{a} \mathrm{A}$ & $40 \mathrm{~cd} \mathrm{~B}$ \\
4 & $92 \mathrm{a} \mathrm{A}$ & $95 \mathrm{a} \mathrm{A}$ & $90 \mathrm{a} \mathrm{A}$ & $82 \mathrm{a} \mathrm{A}$ \\
5 & $90 \mathrm{ab} \mathrm{A}$ & $100 \mathrm{a} \mathrm{A}$ & $95 \mathrm{a} \mathrm{A}$ & $50 \mathrm{~cd} \mathrm{~B}$ \\
7 & $67 \mathrm{~b} \mathrm{~B}$ & $95 \mathrm{a} \mathrm{A}$ & $72 \mathrm{ab} \mathrm{B}$ & $27 \mathrm{~d} \mathrm{C}$ \\
8 & $80 \mathrm{ab} \mathrm{A}$ & $97 \mathrm{a} \mathrm{A}$ & $52 \mathrm{~b} \mathrm{~B}$ & $57 \mathrm{bc} \mathrm{B}$ \\
10 & $95 \mathrm{a} \mathrm{AB}$ & $100 \mathrm{a} \mathrm{A}$ & $95 \mathrm{a} \mathrm{AB}$ & $77 \mathrm{ab} \mathrm{B}$ \\
\hline
\end{tabular}

*Médias seguidas pela mesma letra minúscula na coluna e maiúscula na linha não diferem entre si pelo teste de Tukey a 5\% de probabilidade.

A menor porcentagem de sementes germinadas obtida nos sub-lotes de sementes danificadas ocorreu devido à grande ocorrência de sementes mortas, indicando que danos internos visualizados pela análise radiográfica prejudicam a germinação da semente (Figura 2). Apesar de não diferir estatisticamente, as porcentagens de germinação das sementes do sub-lote cheias foram, em valores absolutos, superiores em relação à testemunha, com incrementos de 3 até $28 \%$.

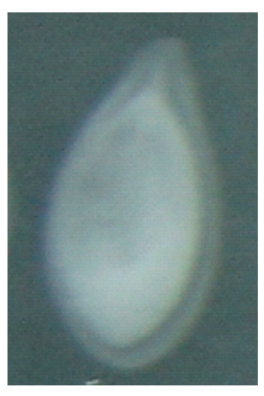

Semente danificada

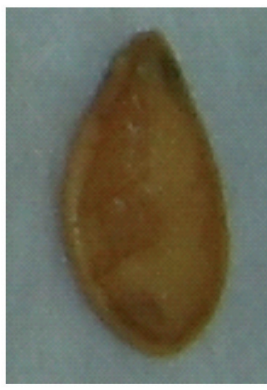

Semente morta

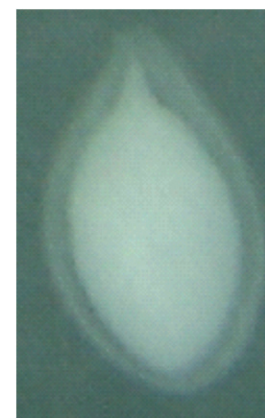

Semente translúcida

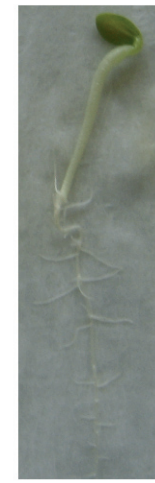

Plântula normal

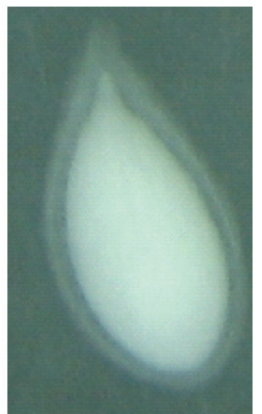

Semente cheia

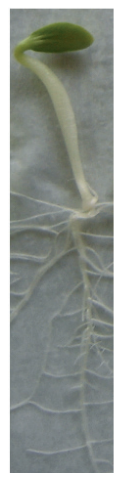

Plântula normal

\section{FIGURA 2. Sementes de abóbora (Cucurbita moschata) classificadas por meio da análise radiográfica de acordo com a} anatomia interna e após o teste de germinação.

Assim como para os resultados de primeira contagem de germinação, não foram observadas diferenças entre os lotes no sub-lote de sementes Cheias no final do teste de germinação, sendo que a presença de sementes danificadas e translúcidas altera a qualidade fisiológica da maioria dos lotes. 
Pela análise dos resultados obtidos, pode ser observado o efeito negativo de sementes danificadas fisicamente na qualidade de lotes de sementes de abóbora, sendo que o descarte de sementes danificadas, visualizadas por meio das radiografias, poderá proporcionar melhoria na qualidade física e consequentemente na qualidade fisiológica dos lotes. Além disso, esses resultados confirmam que a morfologia interna pode ser um indicativo do potencial de viabilidade (Copeland, 1976; Simak et al., 1989; Marcos Filho, 1994)

Resultados semelhantes, indicativos de que sementes danificadas prejudicam o desempenho de lotes de sementes, foram obtidos em trabalhos realizados com espécies florestais como ipê (Oliveira et al., 2004), canafístula (Oliveira et al., 2003) e aroeira-branca (Machado \& Cícero, 2003).

Segundo Carvalho \& Oliveira (2006), a técnica de radiografia não resolve todos os problemas de detecção da qualidade da semente, mas permite diagnose rápida e não destrutiva, na maioria dos casos, fornecendo informações atuais, úteis e essenciais para trabalho científico e controle de qualidade de sementes.

\section{Experimento 2}

A radiação não causou efeito significativo nas variáveis estudadas. Esses resultados indicam que a radiação não influencia na qualidade fisiológica dos lotes de sementes de abóbora antes e após um ano de armazenamento.

Resultados semelhantes foram obtidos por Bino et al. (1993), em sementes de Arabidopsis, que relataram que a pequena dose de radiação usada durante o teste para avaliação da qualidade das sementes não causa danos. No entanto, sementes de soja irradiadas com mais de $50 \mathrm{kV}$ tiveram sua qualidade fisiológica reduzida no decorrer do período de armazenamento com o aumento da intensidade utilizada (Ascheri et al., 2005).

As sementes de abóbora tiveram a velocidade de germinação reduzida devido ao período de armazenamento; porém, o total de plântulas normais no final do teste de germinação não foi alterado pelo tempo de armazenamento e o desempenho das plântulas no teste de emergência foi alterado apenas para sementes que não foram expostas a radiação (Tabela 4).

TABELA 4. Resultados dos testes de Primeira Contagem de Germinação (PCG), Germinação (G), Emergência (E) para sementes de abóbora antes (Época 1) e após o armazenamento (Época 2).

\begin{tabular}{ccccccc}
\hline \multirow{2}{*}{ Radiação } & \multicolumn{2}{c}{ PCG $(\%)$} & \multicolumn{2}{c}{$\mathrm{G}(\%)$} & \multicolumn{2}{c}{ E $(\%)$} \\
\cline { 2 - 6 } & Época 1 & Época 2 & Época 1 & Época 2 & Época 1 & Época 2 \\
\hline \multirow{2}{*}{ Com } & 78 a A & 66 a B & 84 a A & 84 a A & 84 a A & 84 a A \\
Sem & 78 a A & 67 a B & 83 a A & 84 a A & 86 a A & 80 a B \\
\hline
\end{tabular}

* Médias seguidas pela mesma letra minúscula na coluna e maiúscula na linha, não diferem entre si dentro de cada teste pelo teste de Tukey a $5 \%$ de probabilidade.

Como a radiação usada em sementes de abóbora não interfere na sua qualidade e ainda auxilia na identificação de sementes danificadas, que prejudicam a qualidade fisiológica do lote, o teste de raios $\mathrm{X}$ pode ser usado como auxilio na avaliação da qualidade das sementes, possibilitando a obtenção de lotes de qualidade superior.

\section{CONCLUSÕES}

O teste de raios $\mathrm{X}$, na intensidade de $45 \mathrm{kV}$ por 25 segundos, é eficiente para identificar danos internos em sementes de abóbora que afetam negativamente sua qualidade fisiológica.

A exposição das sementes à radiação de $45 \mathrm{kV}$ por 25 segundos, não afeta a qualidade fisiológica das sementes de abóbora mesmo após seu armazenamento em condições controladas pelo período de um ano.

\section{AGRADECIMENTOS}

\section{SEMINIS DO BRASIL, FAPEMIG, CNPQ e FAPESP}

\section{REFERÊNCIAS}

ARGERICH, C.A.; BRADFORD, K.J. The effects of priming and ageing on seed vigour in tomato. Journal Experimental Botany, n.40, p.599-607, 1989.

ASCHERI, D. P. R.; OLIVEIRA NETO, M. C.; DEVILLA, I. A. Qualidade fisiológica de sementes de soja irradiadas 
por raios $\mathrm{X}$ e armazenadas. Revista Brasileira de Armazenamento. v.30, n.2, p 192-197, 2005.

BINO, R.J.; AARTASE, J.W.; VAN DER BURG, W.J. Nondestructive $\mathrm{X}$-ray analysis of Arabidopsis embryo mutants. Seed Science Research, v.3, p.167-170, 1993.

BRASIL. Ministério da Agricultura. Regras para análise de sementes. Brasília, DF: Departamento Nacional de Produção Vegetal, 1992.365p.

CARVALHO, M.L.M.; OLIVEIRA, L.M. Raios X na avaliação da qualidade de sementes. Informativo Abrates, Brasília, DF, v.16, n¹, 2,3, 2006.

COPELAND, L. O. Principles of seed science and technology. Minneapolis: Burges Publishung Company, 1976. 369p.

ISTA. International Rules For Seed Testing Association, Zurich, 174p. 2004.

MACHADO, C.F.; CICERO, S.M. Aroeira-branca (Lithraea molleoides (Vell.) Engl. - Anacardiaceae) seed quality evaluation by the X-ray test. Scientia Agricola, v.60, n.2, p.393-397, Abr./Jun. 2003.

MARCOS FILHO, J. Utilização de testes de vigor em programas de qualidade de sementes. Informativo Abrates, Londrina, v.4, n.2, p. 33-35, 1994.

OLIVEIRA, L.M.; CARVALHO, M.L.M.; GUIMARÃES,
R.M.; MASETTO, T.E. Avaliação da qualidade de sementes de Tabebuia serratifolia Vahl Nich. e T. impetiginosa (Martius ex A. P. de Candolle) Standley pelo teste de raios X. Revista Brasileira de Sementes, v.26, p.138 - 143. 2004.

OLIVEIRA, L.M.; CARVALHO, M.L..M.; DAVIDE, A.C. Utilização do teste de raios-X na avaliação da qualidade de sementes de canafístula (Peltophorum dubium (Sprengel) Taubert. Revista Brasileira de Sementes, v.25, p.116 120. 2003.

SILVA, M. C. P. Teste de tetrazólio em sementes de abóbora (Cucurbita moschata). 2006. 17f. (Monografia lato senso)- UFLA, Lavras.

SIMAK, M.; BERGSTEN, U.; HENRIKSSON, G. Evaluation of ungerminated seeds at the end germination test by radiography. Seed Science and Tecnology, v.17, n.2, p.361-369, 1989.

SIMAK, M. Testing of forest tree and shrub seeds by X-radiography. In: Tree and shrub seed handbook. Chapter 14. International Seed Testing Association. (ISTA), Zurich, Switzerland. 1991.

VAN DER BURG, W.J.; AARTSE, J.W.; VAN ZWOL,R.A; JALINK,H.; BINO, F.J. Predicting tomato seedling morphology by X-ray analysis of seeds. Journal of the American Society for Horticultural Science, v.119, n.2, p.258-263, 1994. 